\title{
Tumores de la región pineal en la edad pediátrica. Presentación de 23 casos y revisión de la bibliografía
}

\author{
Marta Navas-García, Francisco Goig-Revert, Francisco J. Villarejo-Ortega, Javier Robla, Inmaculada de Prada, \\ Luis Madero, Carlos Pérez-Díaz, M. Belén Rivero-Martín, Alfonso Pascual Martín-Gamero, Marcelo Budke, \\ Francisco Cordobés-Tapia
}

Introducción. Los tumores de la región pineal se localizan en una encrucijada de estructuras neurovasculares de difícil acceso quirúrgico y limitadas posibilidades de resección; por ello, el manejo de estas lesiones habitualmente requiere la asociación de tratamiento adyuvante radioterápico y/o quimioterápico.

Pacientes y métodos. Análisis retrospectivo de las características epidemiológicas, clínicas, neurorradiológicas y anatomopatológicas de 23 pacientes con tumores de la región pineal tratados entre los años 1997 y 2010 en el Hospital Infantil Niño Jesús. Se discuten los factores implicados en el pronóstico de esta cohorte tras el tratamiento quirúrgico o adyuvante.

Resultados. El estudio incluyó 6 niñas y 17 niños con edades comprendidas entre los 4 meses y 18 años. El 95\% de los pacientes comenzó con signos de hidrocefalia aguda o subaguda, que precisaron la implantación de una derivación ventriculoperitoneal (82\%). Se obtuvo una muestra histológica tumoral en todos los casos. Cinco pacientes fueron biopsiados y 18 fueron intervenidos mediante craneotomía. El germinoma (ocho casos) y el teratoma maduro (un caso) fueron los tumores con mayor supervivencia; los tumores no germinomatosos (tres casos), del parénquima pineal (cuatro casos) y gliomas (cinco casos) presentaron mayor tasa de recidiva y un peor pronóstico.

Conclusión. El estudio de marcadores tumorales permite orientar el diagnóstico de determinados tumores de la región pineal. Actualmente, se recomienda realizar una toma histológica tumoral para establecer un diagnóstico preciso y un tratamiento oncológico específico.

Palabras clave. Astrocitoma. Germinoma. Glándula pineal. Pineoblastoma. Pineocitoma. Región pineal. Tumor.

\section{Introducción}

La glándula pineal es un órgano derivado del techo del diencéfalo que se desarrolla durante el segundo mes de gestación. Se encuentra delimitada dorsalmente por el esplenio del cuerpo calloso y la tela coroidea, medialmente por la lámina cuadrigémina y el tectum mesencefálico, a nivel anterior por la pared posterior del III ventrículo, y caudalmente por el vermis cerebeloso [1,2]. Esta glándula funciona como un transductor neuroendocrino que sincroniza la liberación hormonal con las fases de luzoscuridad [3].

La región pineal está formada por la glándula pineal y un conjunto de estructuras de origen ependimario (pared del acueducto de Silvio), neural (tálamos y mesencéfalo), glial (tálamos, cuerpo calloso, mesencéfalo), meníngeo (velo interpositum, ápex del tentorio) y vascular (vena de Galeno, venas cerebrales internas, arterias cerebrales posteriores y coroideas posteriores). A consecuencia de la variabilidad histológica de los distintos componentes de la región pineal, existe una amplia representación de tumores que pueden desarrollarse en esta región [1].

En este estudio presentamos una serie formada por 23 pacientes en edad pediátrica que fueron diagnosticados e intervenidos de tumores de la región pineal (TRP) en el servicio de neurocirugía del Hospital Infantil del Niño Jesús. Asimismo, se realiza una revisión bibliográfica sobre la incidencia de estas lesiones y las diferentes opciones de tratamiento médico y quirúrgico de los TRP.

\section{Pacientes y métodos}

Se realiza un estudio descriptivo retrospectivo mediante la revisión de las historias clínicas de una cohorte formada por 23 pacientes en edad pediátrica diagnosticados de TRP. Todos los pacientes fueron intervenidos en el servicio de neurocirugía pediátrica del Hospital Infantil Universitario Niño Jesús
Servicio de Neurocirugía (M. Navas-García, F. Goig-Revert, C. Pérez-Díaz, M.B. Rivero-Martín, A. Pascual Martín-Gamero, M. Budke, F. Cordobés-Tapia): Servicio de Anatomía Patológica (J. Robla); Servicio de Oncología Médica (L. Madero); Hospital Infantil Universitario Niño Jesús; Madrid. Servicio de Neurocirugía; Complejo Asistencial de León; León, España (I. de Prada).

Correspondencia:

Dr. Francisco J. Villarejo Ortega. Servicio de Neurocirugía Pediátrica. Hospital Infantil Universitario Niño Jesús. Avda. Menéndez Pelayo, 65. E-28009 Madrid.

E-mail:

fvillarejo@neuropsico.es

Aceptado tras revisión externa: 01.02.11.

Cómo citar este artículo: Navas-García M, Goig-Revert F, Villarejo-Ortega FJ, Robla J, De Prada I, Madero L, et al. Tumores de la región pineal en la edad pediátrica. Presentación de 23 casos y revisión de la bibliografía. Rev Neurol 2011; 52: 641-52.

(c) 2011 Revista de Neurología 
a lo largo de un período de 13 años (1997-2010). Los criterios de inclusión empleados fueron: pacientes menores de 18 años, con evaluación clínica detallada antes y después de la intervención, y estudios de imagen con resonancia magnética craneal y espinal pre y postoperatoria. Se elaboró un protocolo de recogida de datos que incluía los antecedentes personales y familiares de cada paciente, su sintomatología clínica en el momento del diagnóstico, las características morfológicas y de señal de los diversos TRP en los estudios de neuroimagen, la histología de cada lesión y marcadores tumorales analizados, el tratamiento quirúrgico y coadyuvante realizado, y, finalmente, la evolución postoperatoria y el seguimiento de cada uno de los pacientes.

El análisis de datos incluyó las variables de edad, sexo, signos y síntomas de inicio, presencia de hidrocefalia y necesidad de implantación de válvula de derivación ventriculoperitoneal, aumento de marcadores tumorales en el líquido cefalorraquídeo (LCR) y sangre, diagnóstico anatomopatológico del tumor, tipo de abordaje quirúrgico, grado de resección, tratamiento con quimioterapia y/o radioterapia, tiempo de seguimiento, y estado clínico y supervivencia de los pacientes a lo largo de su seguimiento.

Se realizó, asimismo, una revisión sistemática de la bibliografía, empleando la base de datos Medline y seleccionando los artículos publicados más recientemente que incluían información esencial acerca del diagnóstico y el manejo terapéutico de los TRP.

\section{Resultados}

La tabla I resume la información clínica obtenida de los 23 pacientes incluidos en el estudio. Seis niñas y 17 niños fueron incluidos en el análisis, constatándose un claro predominio de TRP en el sexo masculino (74\%). La edad media de los pacientes fue 10,9 años, con un valor mínimo de 4,5 meses y un máximo de 18 años. Los síntomas referidos por los pacientes de forma mayoritaria fueron cefalea (el 79\% de los pacientes), náuseas y vómitos (56\%) y síntomas visuales, como diplopía secundaria, y parálisis de pares craneales (43\%). Se evidenció la existencia de hidrocefalia obstructiva en los estudios de neuroimagen en 22 de los 23 pacientes. Dos casos cursaron con alteraciones neuroendocrinas (diabetes insípida y pubertad precoz), como cuadro clínico de presentación del tumor pineal. Se confirmó la presencia del signo de Parinaud como inicio clínico en 9 de los 23 pacientes estudiados, debido a la compresión de la placa cuadrigeminal mesencefálica. En cinco pacientes se observó edema de papila en el examen del fondo de ojo y dos niños presentaron un aumento del perímetro craneal (macrocefalia) en el momento del ingreso. Todos los pacientes fueron estudiados con tomografía axial computarizada (TAC) y resonancia magnética (RM) cerebral. El tamaño medio de las lesiones fue de $3,2 \mathrm{~cm}$. El $74 \%$ de los tumores correspondió a masas sólidas en el estudio de neuroimagen, y en los cinco casos restantes las lesiones presentaron características mixtas de masa sólido-quística.

El diagnóstico histológico del tumor se obtuvo mediante biopsia en cinco pacientes (tres mediante técnica estereotáctica y dos mediante endoscopia) y a través de craneotomía y abordaje supracerebeloso infratentorial en el resto de los casos (78\%). Entre estos últimos tumores intervenidos, se logró una resección tumoral completa en seis pacientes (un pineoblastoma, un astrocitoma II-III, un teratoma maduro, un pineocitoma, un ependimoma y un quiste pineal), mientras que la resección fue subtotal en los otros 12 casos. El diagnóstico anatomopatológico más frecuente en esta serie fue el de germinoma (ocho pacientes), seguido del pineocitoma (dos casos), pineoblastoma (dos casos), teratoma inmaduro (dos casos) y astrocitoma de grado intermedio (dos casos). En todos se cuantificaron los niveles de marcadores tumorales en el suero y el LCR; los valores de alfafetoproteína se encontraron elevados en dos pacientes (un teratoma maduro y un teratoma inmaduro) y se demostró un incremento de la fracción beta de la gonadotropina coriónica en otros tres casos (un germinoma, un tumor de seno endodérmico y un teratoma inmaduro). Se confirmó la diseminación leptomeníngea tumoral mediante estudio citológico del LCR para células malignas en tres pacientes (dos germinomas y un pineoblastoma).

Tras el tratamiento quirúrgico un total de 13 pacientes (56\%), recibieron radioterapia adyuvante con una dosis media de 49,5 Gy (rango: 43-59 Gy) en el lecho tumoral. Once niños (48\%) recibieron tratamiento quimioterápico adicional, siguiendo distintos protocolos farmacológicos específicos basados en la estirpe tumoral de cada paciente. La complicación postoperatoria más grave fue una hemorragia del lecho quirúrgico en un paciente intervenido de un germinoma, y la neutropenia febril fue la complicación más grave asociada al tratamiento con quimioterapia, que condicionó el fallecimiento por sepsis en tres casos. Se monitorizó la evolución de todos los pacientes mediante revisiones periódicas tanto en la consulta de neurocirugía como en la consulta de oncología médica del Hospital Niño Jesús; el seguimiento medio fue de 36 meses, siendo la principal causa de abandono el fallecimiento del paciente. 


\section{Discusión}

\section{Aproximación histórica y epidemiología de los TRP}

El tratamiento quirúrgico de los TRP clásicamente se ha considerado un reto para el neurocirujano, debido a los pobres resultados obtenidos y a las elevadas cifras de mortalidad descritas en algunas series, con porcentajes cercanos al 90\%, a principios del siglo xx. Horsley realizó el primer intento de escisión quirúrgica de una lesión pineal en 1910, pero Krause describió por primera vez el abordaje supracerebeloso infratentorial para la resección de los TRP en 1926. Posteriormente, en 1936, Dandy realizó el primer abordaje interhemisférico para la resección de un tumor en esta localización. Debido a las elevadas cifras de morbimortalidad observadas con el tratamiento quirúrgico de los TRP, Torkildsen propuso en 1948 el tratamiento conservador de estos tumores mediante derivación del LCR y radioterapia, reservando la extirpación quirúrgica exclusivamente para los TRP radiorresistentes $[1,4]$.

El diseño de nuevos abordajes quirúrgicos, las mejoras técnicas anestésicas y el desarrollo de microscopios quirúrgicos redujeron de forma significativa las tasas de morbimortalidad asociadas a la cirugía de los TRP en las décadas de los setenta y ochenta. La incorporación de las técnicas microquirúrgicas al abordaje supracerebeloso infratentorial fue promovida por Stein a principios de la década de los setenta, y permitió reducir de forma notoria las complicaciones intraoperatorias de este tipo de intervención. En consecuencia, en las últimas décadas se ha sustituido la aplicación de tratamiento radioterápico empírico de estas lesiones por una decisión terapéutica basada en el diagnóstico histológico obtenido mediante biopsia o resección de la masa tumoral $[1,2,4]$.

Los TRP representan en la actualidad el 0,4-1\% de los tumores intracraneales en adultos y el 3-8\% en la edad pediátrica [5]. Son más frecuentes en niños entre 1-12 años, donde constituyen alrededor del 3\% de los tumores cerebrales [6]. En los adultos, estas lesiones suelen diagnosticarse a partir de la tercera década. Los TRP son más frecuentes en el sexo masculino ( 3 a 1); en concreto, los tumores de células germinales son 12 veces más frecuentes en varones [5]. De acuerdo con la bibliografía, en nuestra serie constatamos una incidencia tres veces superior de TRP en niños respecto a niñas.

\section{Presentación clínica de los TRP}

Desde el punto de vista fisiopatológico, la sintoma- tología clínica producida por los TRP está directamente relacionada con la localización específica del tumor, que produce un efecto compresivo sobre estructuras neurales adyacentes. La infiltración local de las diferentes estructuras neurovasculares de la región pineal también puede contribuir al desarrollo de la sintomatología. La tasa y la velocidad de crecimiento tumoral determinan la rapidez con la que se instauran los diversos síntomas y constituyen un factor pronóstico importante en estos pacientes [1].

La tríada clásica del síndrome de hipertensión intracraneal, cefalea, náuseas, vómitos y papiledema, está presente hasta en el $70 \%$ de los pacientes con TRP $[1,2,4]$, y se observa con mayor frecuencia en la edad infantil y adolescente que en los adultos. La hipertensión intracraneal habitualmente es secundaria al desarrollo de hidrocefalia obstructiva, secundaria al obstáculo para la circulación del LCR en la parte posterior del III ventrículo y acueducto de Silvio, que determina la expansión tumoral sobre estas estructuras. En nuestra serie, el 95\% de los pacientes presentaba hidrocefalia obstructiva al ingreso. En los lactantes, la hipertensión intracraneal se manifiesta por macrocefalia y aumento del perímetro craneal por encima de los percentiles estándar y, secundariamente, por el abombamiento a tensión de la fontanela anterior, así como por signos de irritabilidad, somnolencia y vómitos en escopetazo que pueden aparecer en estos pacientes. La presencia de ataxia y dismetría es secundaria a la afectación de las fibras cerebelosas eferentes del pedúnculo cerebeloso superior [2].

Henri Parinaud describió a finales del siglo XIX un hallazgo clínico casi constante en pacientes con TRP, consistente en la limitación en la mirada vertical, alteraciones del reflejo pupilar ante la luz, con acomodación conservada y fallo en la convergencia de la visión, como consecuencia de la afectación del colículo superior y el área pretectal del mesencéfalo. Este signo se considera patognomónico de lesiones que afectan la lámina cuadrigémina y está presente en más del $75 \%$ de los pacientes con TRP. La compresión tumoral de la sustancia gris periacueductal puede producir midriasis y nistagmo convergente. Asimismo, se han descrito alteraciones de la agudeza visual, que pueden deberse a la existencia de edema de papila, visible en el fondo de ojo, o bien por diplopía secundaria a la paresia del VI par por los efectos de la hipertensión intracraneal [7].

Este grupo de tumores puede precipitar una disfunción neuroendocrina, debido al efecto de la hidrocefalia o la asociación concurrente de una tumoración supraselar, que se puede manifestar clínicamente como diabetes insípida. Sin embargo, 
Tabla I. Pacientes intervenidos de tumores de la región pineal.

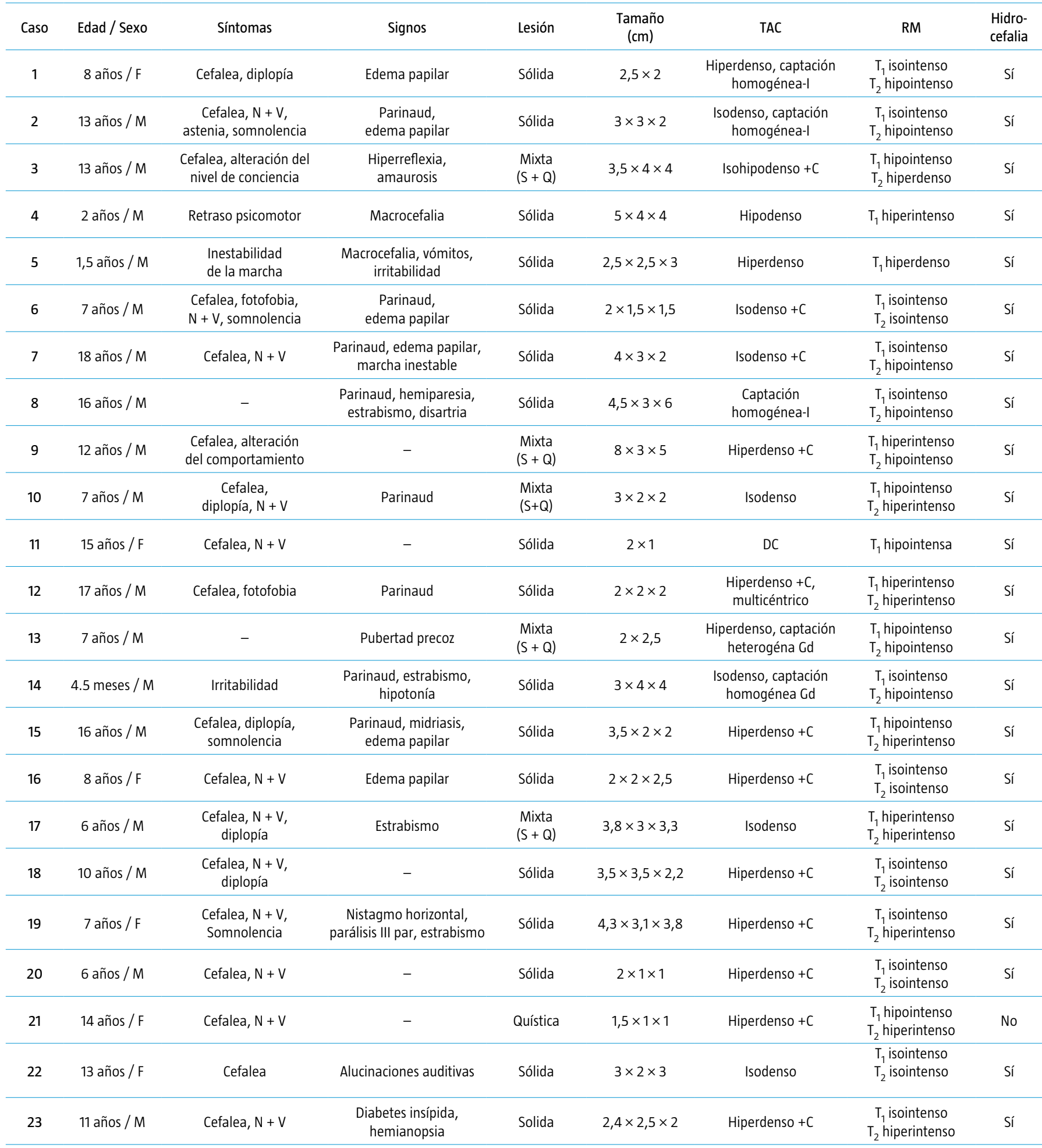

+C: tumor con calcificaciones en estudio de TAC; a-FP: alfafetoproteína; B: biopsia; BEP: bleomicina, VP-16, CDP; bhCG: fracción beta de gonadotropina coriónica; C. meníngea: carcinomatosis meníngea; CDDP: cisplatino; DVP: derivación ventriculoperitoneal; E: fallecimiento; F: femenino; LCR: líquido cefalorraquídeo; M: masculino; N + V: náuseas y vómitos; Q: quística; R-FC: ci- 


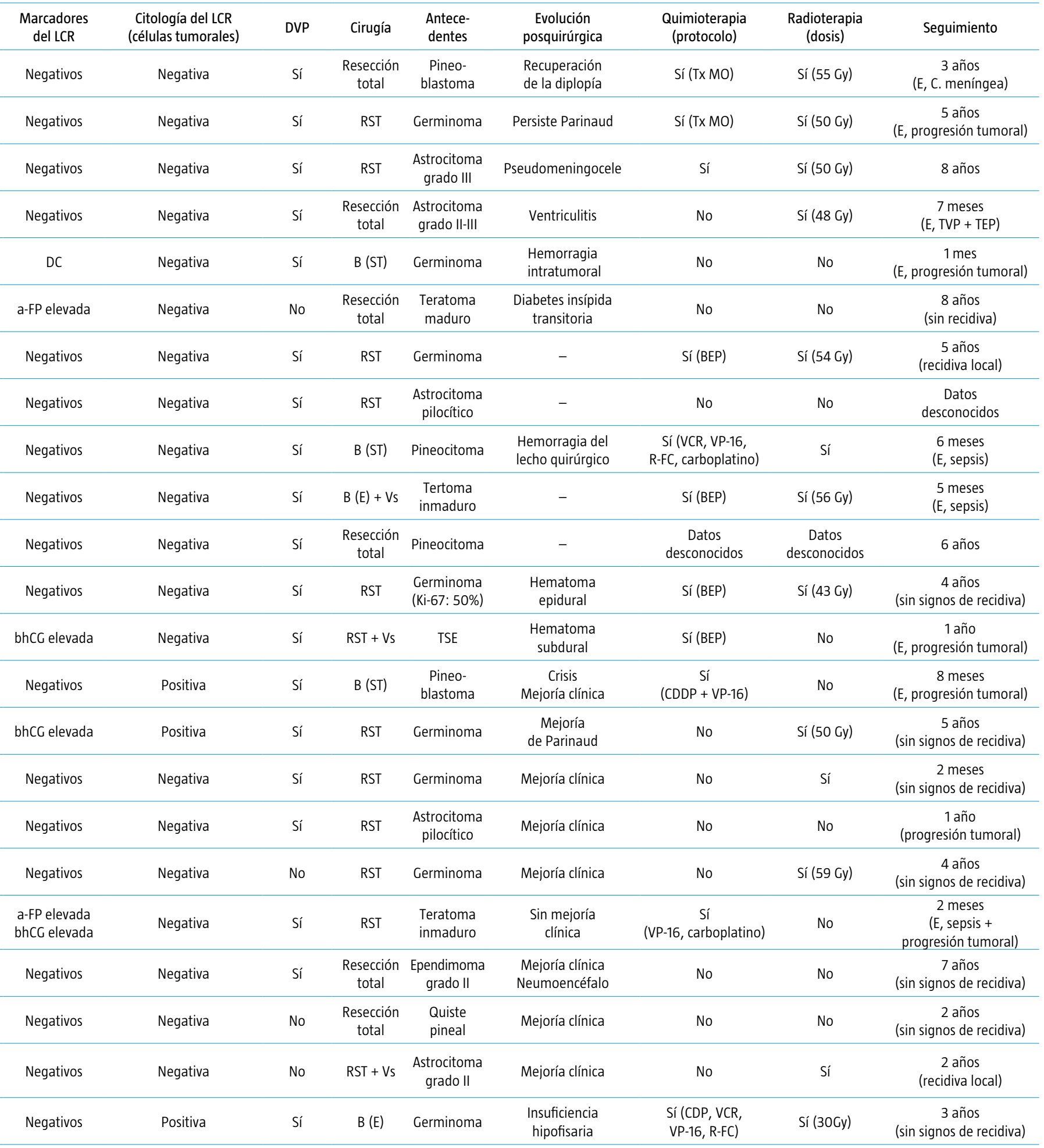

clofosfamida; RM: resonancia magnética; RST: resección subtotal; S: sólida; TAC: tomografía axial computarizada; TSE: tumor del seno endodérmico; Tx MO: trasplante de médula ósea; VCR: vincristina; VP-16: etopósido; Vs: ventriculostomía. 
esta sintomatología habitualmente se produce por invasión hipotalámica o del receso anteroinferior del III ventrículo, o bien por afectación hipofisaria secundaria a la extensión directa de la masa o a su diseminación leptomeníngea. En nuestra casuística, dos pacientes diagnosticados de un tumor de seno endodérmico y un germinoma cursaron, respectivamente, con pubertad precoz y diabetes insípida. Otros síndromes endocrinos más específicos, como la pseudopubertad precoz o el hipogonadismo, pueden surgir por la secreción hormonal de gonadotropina coriónica humana (bhCG) por las células germinales tumorales. Se ha descrito amenorrea secundaria en el 93\% de las niñas por encima de los 12 años con TRP, y detención del crecimiento en el $33 \%$ de los pacientes menores de 15 años con tumores de células germinales (TCG) [2,5].

El sangrado intratumoral, o apoplejía pineal, se ha descrito como una manifestación clínica de presentación poco frecuente entre los TRP. A pesar de que el sangrado preoperatorio espontáneo de tumores hipervascularizados en esta localización está descrito en la bibliografía, su incidencia es baja y es más habitual que ocurra como complicación postoperatoria [1].

\section{Clasificación histológica de los TRP}

De forma general, los TRP pueden clasificarse como TCG (60\%), tumores derivados del parénquima pineal (TPP) (30\%) y gliomas (5\%), derivados, respectivamente, de tejidos embrionarios ectópicos, de las células del parénquima pineal, o bien de la astroglía circundante [5]. Otros tumores que pueden desarrollarse en la región pineal son meningiomas, gangliogliomas, ependimomas, lipomas o metástasis, con una frecuencia muy inferior a los primeros. Por otro lado, los quistes pineales, las malformaciones vasculares y los aneurismas de la vena de Galeno son lesiones benignas situadas en la región pineal que pueden ser confundidas con TRP [8].

Los TCG derivan de células pluripotenciales germinativas de estructuras de línea media. Son los tumores más frecuentes de la región pineal (50-75\%), con una mayor incidencia en el sexo masculino. Se clasifican en germinomas - grado II de la Organización Mundial de la Salud (OMS) - y tumores no germinomatosos, estos últimos representados por el teratoma maduro (grado I) o inmaduro (grado III-IV), el coriocarcinoma, el carcinoma embrionario y el tumor del seno endodérmico. Tanto el coriocarcinoma, como el tumor del seno endodérmico y el carcinoma embrionario se clasifican como un grado biológico IV de agresividad tumoral.
Los germinomas son globalmente las neoplasias más frecuentes de la región pineal (45-50\%), y constituyen el $75 \%$ de todos los tumores derivados de células germinales [5]. La mayoría de los pacientes se diagnostica dentro de la tercera década, con un pico de incidencia alrededor de los 20 años, y su incidencia es mayor en el sexo masculino (13 a 1). La localización pineal es más frecuente en el sexo masculino, mientras que los germinomas ectópicos supraselares son habitualmente diagnosticados en niñas. Dado que estos tumores carecen de cápsula, pueden invadir estructuras adyacentes y diseminarse fácilmente a través del LCR a nivel subependimario y espinal.

El teratoma es el segundo tumor germinal más frecuente (15\%). Está formado por la transformación tumoral de tejidos primitivos residuales (ectodermo, mesodermo o endodermo), y cada subtipo tumoral representa la correlación maligna de una fase diferente del desarrollo embrionario. En función del grado de diferenciación celular, se clasifican en teratomas maduros (Fig. 1) e inmaduros, y en teratomas con diferenciación maligna. El coriocarcinoma se origina a partir de la malignización de tejido extraembrionario trofoblástico, y su incidencia es mayor durante la primera década de la vida. Pueden presentar hemorragias masivas. Las formas puras son más raras en este tipo tumoral, y generalmente está asociado a germinomas y teratomas inmaduros. Los tumores del seno endodérmico son poco frecuentes (un 3\% de los TRP en niños) y proceden de la transformación maligna de tejido extraembrionario del saco vitelino; las células tumorales son positivas para alfafetoproteína.

Aproximadamente el 20\% de los TRP se origina a partir de las células epiteliales del parénquima de la glándula pineal, denominándose TPP. Histológicamente se clasifican en pineocitoma (OMS grado I), pineoblastoma (OMS grado IV), tumores de diferenciación intermedia y tumor papilar de la región pineal (OMS grado II-III) [9]. En estudios inmunohistoquímicos, son positivos para enolasa específica neuronal y sinaptofisina, marcadores que reafirman su origen neuroendocrino.

El pineocitoma es un tumor habitualmente bien delimitado, más frecuente en adultos jóvenes, sin predilección sexual, y constituye aproximadamente el 45\% de los TPP (Fig. 2). El pineoblastoma es un tumor muy agresivo e infiltrativo, con bordes mal definidos, que afecta con más frecuencia a pacientes en las dos primeras décadas de vida. Representa el $45 \%$ de los TPP y tiene un riesgo muy elevado de diseminación extracraneal y leptomeníngea. Los tumores de diferenciación intermedia constituyen el 
$10 \%$ restante de los tumores del parénquima pineal. Por último, el tumor papilar de la región pineal es una entidad neuroepitelial recientemente añadida al grupo de los TPP que carece de criterios histológicos específicos para definirlo; microscópicamente es indistinguible del pineocitoma, pero ultraestructuralmente posee diferenciación ependimaria procedente del órgano subcomisural. Por ello, su diagnóstico es complejo y es preciso realizar el diagnóstico diferencial entre dicho tumor con el ependimoma papilar y los tumores del plexo coroideo [9].

Aunque las metástasis extracraneales no son frecuentes, la incidencia de diseminación leptomeníngea de los TRP es significativa, variable en función de la histología tumoral; el 19\% de los pineoblastomas y el $12 \%$ de los tumores de células germinales presentan signos de diseminación a través del LCR en el momento del diagnóstico de la lesión $[7,10]$.

\section{Marcadores tumorales de los TRP}

La determinación en el suero y en el LCR de marcadores tumorales es una evaluación preoperatoria de gran utilidad. De forma conjunta con los estudios de neuroimagen, los resultados pueden sugerir la estirpe tumoral, pero sólo ocasionalmente permiten obtener un diagnóstico de certeza. En general, se considera que los tumores secretores tienen un comportamiento más agresivo y asocian un peor pronóstico en comparación con los no secretores. La determinación de marcadores tumorales en el suero y el LCR también es útil para monitorizar la respuesta al tratamiento de estos tumores. En general, las determinaciones en el LCR poseen mayor sensibilidad que las séricas, y el resultado de los marcadores tumorales obtenidos mediante una punción lumbar del LCR (en ausencia de contraindicación para realizarla) se considera más preciso que el obtenido en el sistema ventricular.

Los marcadores tumorales son especialmente útiles en el estudio diagnóstico de los pacientes con TCG, tumores que mantienen características moleculares de su origen embrionario y que, en ocasiones, pueden expresar proteínas como la alfafetoproteína y bhCG. La elevación de estas proteínas indica habitualmente una estirpe germinal maligna. La alfafetoproteína es una glucoproteína cuyos niveles están elevados de forma importante en los tumores del seno endodérmico, y también resultan elevados, aunque en menor cuantía, en el carcinoma embrionario. Aunque, en general, los teratomas no secretan alfafetoproteína, los teratomas menos diferenciados e inmaduros pueden producir pequeñas cantidades detectables en el LCR. Los valores
Figura 1. a) Imagen axial correspondiente al estudio de tomografía axial computarizada realizado en un paciente afecto de teratoma maduro de la región pineal (caso 6; Tabla I). Dicha tumoración presenta calcificaciones en su interior y condiciona la existencia de hidrocefalia obstructiva, por lo que el paciente precisó la colocación de una derivación ventriculoperitoneal. b,c) Sección axial $T_{2}$ y sagital $T_{1}$ del estudio de resonancia magnética, en las que se observa un comportamiento hiperintenso de la lesión en ambas secuencias. d-f) Fotografías intraoperatorias correspondientes a la resección tumoral completa practicada a través de un abordaje supracerebeloso e infratentorial, con exposición de ambos hemisferios cerebelosos (d). Durante la resección microquirúrgica se identificaron ambas venas basales de Rosenthal (R) y la vena de Galeno (G) como estructuras vasculares limitantes del tumor (T).

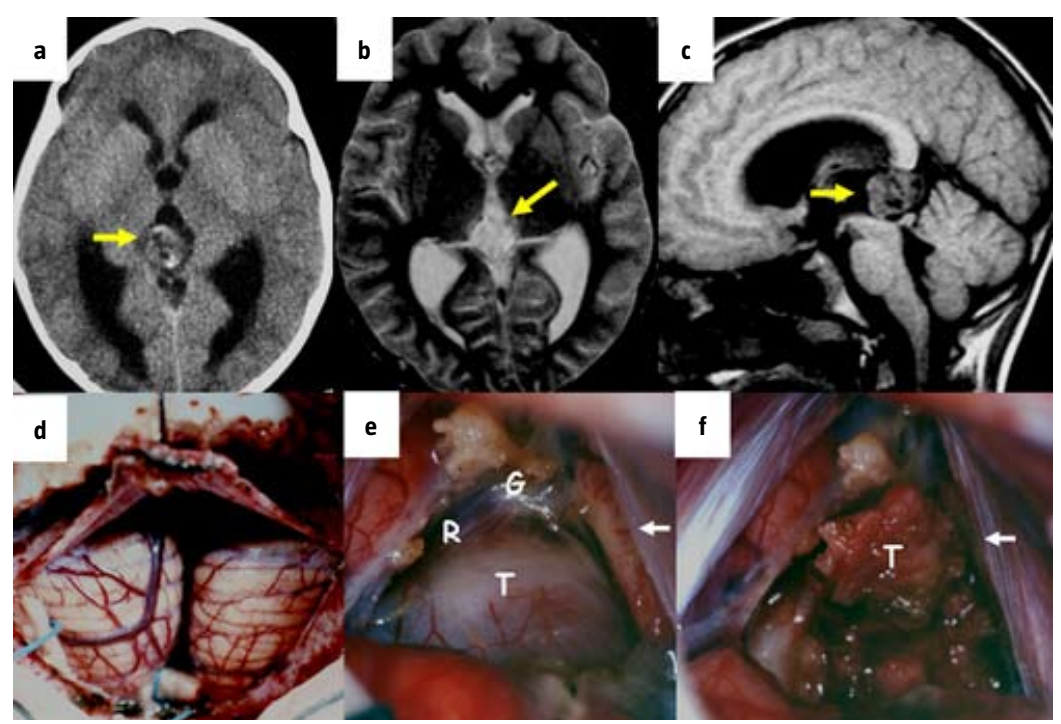

de bhCG se encuentran elevados de forma significativa en el coriocarcinoma. Por otro lado, la presencia de células gigantes sincitiotrofoblásticas en germinomas mixtos pueden ocasionar un aumento en los niveles de esta proteína, pero normalmente los germinomas no la producen. Se consideran positivos valores superiores a $10 \mu \mathrm{g} / \mathrm{L}$ de alfafetoproteína y 50 UI/L de bhCG en el LCR $[1,2,4]$.

\section{Diagnóstico neurorradiológico}

En el estudio de los TRP, es fundamental la realización de pruebas de neuroimagen para evaluar sus características morfológicas y de señal, tamaño, vascularización y las relaciones anatómicas que muestran con las estructuras anatómicas circundantes.

Los pineocitomas suelen ser tumores de pequeño tamaño $(<3 \mathrm{~cm})$, típicamente isodensos, y se realzan de forma homogénea tras la administración de contraste en el estudio de TAC. Existen quistes y calcificaciones, generalmente periféricas, en más de la mitad de los casos. Son lesiones bien delimitadas, hipoisointensas en secuencias potenciadas en $T_{1}$ de 
Figura 2. a,b) Sección axial y sagital $T_{1}$ del estudio de tomografía axial computarizada y resonancia magnética cerebral que fueron realizados en una paciente de 15 años de edad, diagnosticada de pineocitoma de la región pineal (caso 11; Tabla I). Se observó una tumoración sólida, de gran tamaño, sin una delimitación clara, con calcificaciones centrales (flecha) e hidrocefalia obstructiva asociada. c,d) La paciente fue intervenida mediante un abordaje supracerebeloso e infratentorial a la tumoración, la cual se encontraba íntimamente adherida a la cara basal del tentorio y las venas de Galeno y de Rosenthal (c). Detalle microquirúrgico del componente cálcico de la lesión (C) en íntima relación con las estructuras vasculares venosas de la región pineal.

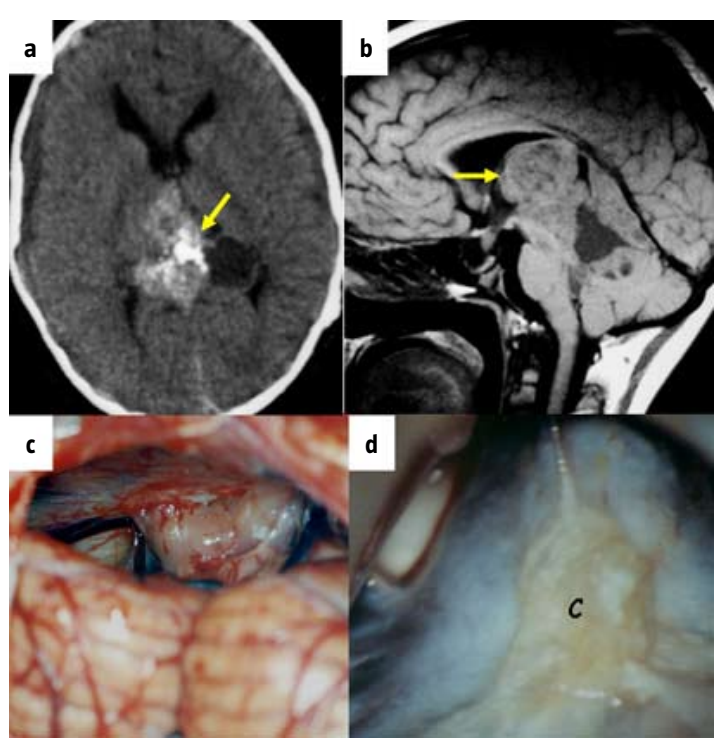

RM, hiperintensas en secuencias potenciadas en $\mathrm{T}_{2}$, y presentan realce homogéneo tras la administración de gadolinio. Los pineoblastomas suelen tener una forma más irregular, peor delimitados, un tamaño considerable (algunos $>4 \mathrm{~cm}$ ) y carecen de calcificaciones (Figs. 3d, 3e y 3f). Son isointensos en secuencias $\mathrm{T}_{1}$, realzan de forma heterogénea con contraste, y el $90 \%$ asocia hidrocefalia [11].

Los tumores de origen glial tienen una apariencia radiológica similar en la TAC y la RM a la que presentan en otras localizaciones cerebrales. Pueden contener calcificaciones, situadas con mayor frecuencia en su porción apical, hasta en dos tercios de los pacientes; suelen ser hipointensos en secuencias potenciadas en $T_{1}$ e hiperintensos en las potenciadas en $\mathrm{T}_{2}$, aunque, por lo general, el patrón de realce es muy variable [11].

Los germinomas se presentan como masas bien circunscritas y relativamente homogéneas, mostrándose isodensos o ligeramente hiperdensos en la TAC. La RM cerebral muestra lesiones isointensas en $\mathrm{T}_{1}$, ligeramente hiperintensas en $\mathrm{T}_{2}$, con realce homogéneo e intenso tras la administración de gadolinio. Las calcificaciones, cuando existen, se disponen alrededor de la glándula pineal [11]. Los teratomas son tumores bien delimitados que se caracterizan por su heterogeneidad, multilocularidad y su realce irregular, en ocasiones anular. En determinados casos presentan áreas de baja atenuación, que se correlacionan con la existencia de tejido adiposo, dato útil para el diagnóstico diferencial con otros TRP. La presencia de hemorragias intratumorales puede distinguir subtipos específicos, como el coriocarcinoma. El estudio con arteriografía cerebral selectiva se emplea casi exclusivamente en aquellos pacientes en los que es preciso realizar un diagnóstico diferencial con malformaciones vasculares de la región pineal.

\section{Tratamiento de los TRP}

La glándula pineal es una estructura cerebral medial profunda, rodeada de una importante red vascular arterial y venosa y de estructuras centroencefálicas vitales; por este motivo, el abordaje quirúrgico de los TRP es de alta complejidad y tradicionalmente ha constituido un reto para el neurocirujano.

\section{Tratamiento quirúrgico}

Dado que el tratamiento específico de los TRP viene determinado por su histología, es fundamental la obtención de tejido tumoral mediante un abordaje quirúrgico de la lesión previamente al inicio de tratamiento coadyuvante con radioterapia o quimioterapia [5]. No obstante, en determinados casos de tumores no germinomatosos (teratomas maduros) con marcadores tumorales elevados (alfafetoproteína y bhCG) y estudios de neuroimagen compatibles, puede ser razonable iniciar tratamiento empírico (radioterapia y/o quimioterapia) sin necesidad de realizar una biopsia tumoral previa $[1,12]$. La elección del abordaje quirúrgico más apropiado en cada caso depende de la experiencia y las aptitudes del neurocirujano, puesto que existen ventajas y desventajas específicas para los distintos procedimientos.

La realización de una craneotomía, con exposición y resección tumoral completa de la tumoración, se considera por algunos autores como el tratamiento quirúrgico de elección en pacientes con TRP [13]. La cirugía abierta con el empleo de las técnicas microquirúrgicas disponibles hoy en día pretende conseguir la toma de biopsia, o bien la resección total o subtotal de la tumoración; se considera un método de tratamiento efectivo y seguro, a 
pesar de que las cifras de morbimortalidad publicadas en la actualidad se sitúan entre el 3-10\% y el 4-10\%, respectivamente $[1,2,5]$. En la bibliografía se han descrito distintas vías de acceso a la región pineal, dependiendo de las características individuales del paciente y del tumor; en la tabla II se sintetizan las ventajas y desventajas asociadas a cada uno de dichos abordajes quirúrgicos. Puesto que la tercera parte de los TRP son benignos, la cirugía abierta puede ser potencialmente curativa, como en el caso de los teratomas maduros, sin la necesidad de asociar tratamientos adyuvantes. La evidencia científica sugiere que los pacientes afectos de tumores malignos se benefician del vaciamiento quirúrgico de la lesión, puesto que éste puede mejorar la respuesta al tratamiento coadyuvante [13-15].

La biopsia endoscópica es un método quirúrgico de diagnóstico para la obtención de tejido tumoral, que obvia la necesidad de cirugía abierta, dependiendo de la habilidad y la experiencia del cirujano. Algunos trabajos han publicado un rendimiento diagnóstico con esta técnica del 94\%, aunque algunos pacientes necesitan un segundo tiempo quirúrgico [16]. Permite la obtención de LCR para el estudio de marcadores tumorales, la valoración del grado de infiltración tumoral mediante visualización del III ventrículo, y la realización de una ventriculostomía en casos con hidrocefalia asociada [16].

En aquellos pacientes con múltiples patologías, enfermedad ampliamente diseminada o con la sospecha de tumoración maligna claramente invasiva, la biopsia estereotáctica a través de un abordaje frontal inferior a las venas cerebrales internas se ha definido como el procedimiento diagnóstico de elección. Esta técnica puede realizarse de forma segura y eficaz en centros especializados, pero supone una desventaja para aquellos pacientes que podrían beneficiarse de una resección tumoral total o subtotal. Regis et al publicaron en su trabajo una mortalidad del $1,3 \%$ y una morbilidad inferior al $1 \%$, asociadas a esta técnica como consecuencia del riesgo de dañar estructuras vasculares del sistema venoso profundo. La tasa de diagnóstico histológico lograda con la biopsia estereotáctica se encuentra cercana al 94-100\% cuando se toman varias muestras tumorales $[17,18]$.

En general, tanto la biopsia endoscópica como la estereotáctica están sujetas a posibles errores diagnósticos, debido a la heterogeneidad de algunos tumores pineales (fundamentalmente, los tumores de células germinales mixtos). Aunque se ha descrito un amplio grupo de complicaciones, la morbilidad más frecuente es un empeoramiento transitorio y autolimitado de los síntomas oculares [17-20].
Figura 3. a) Imagen axial de una tumoración de la región pineal hiperdensa en el estudio de tomografía axial computarizada cerebral con contraste, correspondiente a un tumor de seno endodérmico en un paciente de 7 años (caso 13; Tabla I). b) La sección sagital $\mathrm{T}_{1}$ con contraste del estudio de resonancia magnética cerebral mostró una gran tumoración hiperintensa sólido-quística alojada en la región pineal. c) A pesar de una resección tumoral parcial y el tratamiento coadyuvante postoperatorio, la lesión presentó una importante progresión tumoral evidenciada en estudio de tomografía axial computarizada cerebral, que condicionó el fallecimiento del paciente un año después del diagnóstico inicial. d-f) Secciones axial, coronal y sagital del estudio de resonancia magnética cerebral realizado en un paciente de 4 meses de edad, de una lesión sólido-quística hiperintensa en secuencias $T_{1}$ y $T_{2}$, compatible con pineoblastoma de la región pineal (caso 14; Tabla I). La tumoración fue biopsiada y tratada con quimioterapia adyuvante postoperatoria debido a la edad del paciente, el cual falleció ocho meses después del diagnóstico inicial.

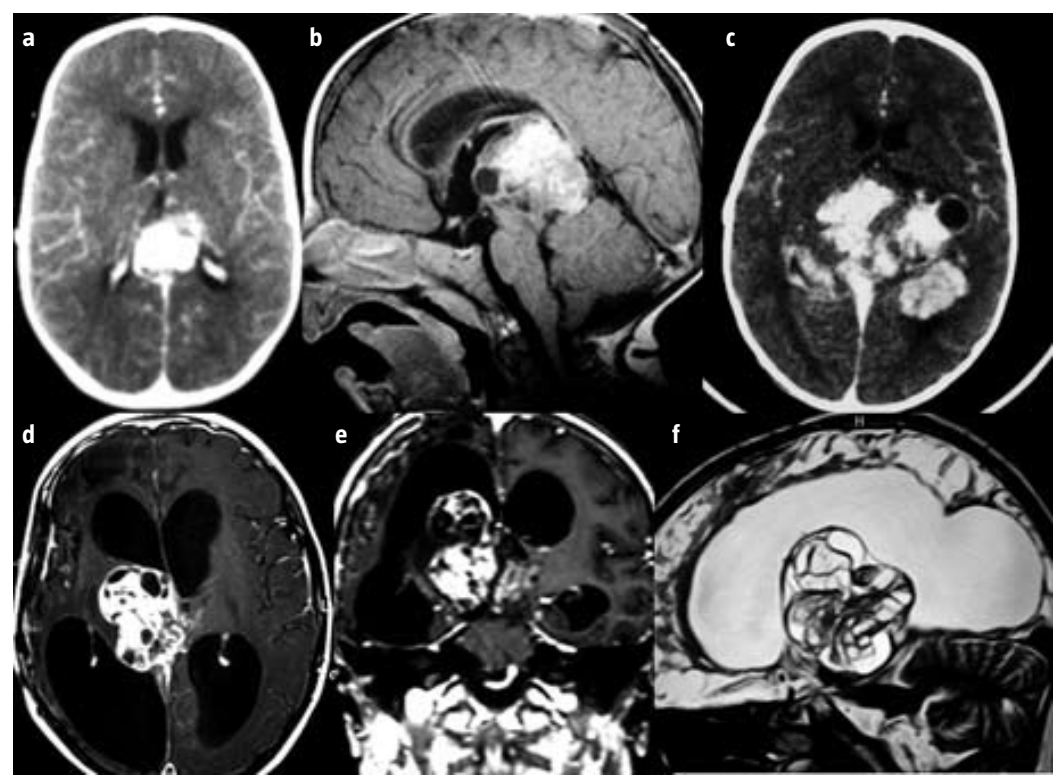

Cuando el diagnóstico histológico de la lesión no es congruente con los hallazgos de marcadores tumorales del LCR y el suero, el tratamiento debe adaptarse al resultado asociado con el tumor más agresivo y de peor pronóstico [1].

\section{Tratamiento adyuvante: \\ radioterapia y quimioterapia}

La incidencia de trastornos neurocognitivos diferidos y endocrinos constituye el principal aspecto negativo del tratamiento con radioterapia holocraneal y/o craneoespinal; dichos efectos deletéreos se ven acentuados en los pacientes de más corta edad.

En los TPP, el tratamiento con radioterapia se recomienda para reducir la tasa de recidiva local en tumores malignos con elevada probabilidad de diseminación leptomeníngea y afectación metastásica a distancia, fundamentalmente los pineoblastomas y algunos tumores con diferenciación intermedia. El control local de la enfermedad es dependien- 
Tabla II. Características de los principales abordajes quirúrgicos a la región pineal.

\begin{tabular}{|c|c|c|}
\hline & Ventajas & Inconvenientes \\
\hline $\begin{array}{l}\text { Supracerebeloso } \\
\text { infratentorial (ASCIT) }\end{array}$ & $\begin{array}{l}\text { Orientación sencilla, abordaje de la línea media } \\
\text { Extraaxial al III ventrículo } \\
\text { Acceso inferior al sistema venoso profundo } \\
\text { Minimiza la lesión de las estructuras nerviosas vitales } \\
\text { Menor morbilidad posquirúrgica } \\
\text { Sin corticotomía occipital o parietal }\end{array}$ & $\begin{array}{l}\text { Campo quirúrgico muy limitado } \\
\text { Coagulación de venas puente laterales y/o vena cerebelosa precentral } \\
\text { Posible lesión del vermis superior } \\
\text { Pobre visualización de estructuras supratentoriales } \\
\text { No apto para lesiones paramedianas del III ventrículo } \\
\text { Visualización inadecuada de la pared posterior del III ventrículo }\end{array}$ \\
\hline $\begin{array}{l}\text { Occipital } \\
\text { transtentorial (AOT) }\end{array}$ & $\begin{array}{l}\text { Campo quirúrgico amplio } \\
\text { Ausencia de venas desde el lóbulo occipital al seno longitudinal superior } \\
\text { Visualización adecuada del sistema venoso profundo } \\
\text { Abordaje extraaxial sobre tentorio } \\
\text { Visualización óptima de la porción dorsal y lateral del tronco ipsilateral }\end{array}$ & $\begin{array}{l}\text { Posibilidad de daño del lóbulo occipital y sistema venoso profundo } \\
\text { Posibles variaciones anatómicas tentoriales } \\
\text { Difícil visualización de la región cuadrigémina y tálamo ipsilateral } \\
\text { Posible necesidad de callosotomía }\end{array}$ \\
\hline $\begin{array}{l}\text { Transcalloso } \\
\text { posterior (ATP) }\end{array}$ & $\begin{array}{l}\text { No depende del tamaño ventricular } \\
\text { Abordaje extraaxial sobre el tentorio } \\
\text { No precisa corticotomía } \\
\text { Posible exposición completa del III ventrículo }\end{array}$ & $\begin{array}{l}\text { Posibilidad lesión de las venas parietales puente } \\
\text { Requiere retracción parietal y callosotomía } \\
\text { Riesgo de daño de las estructuras venosas profundas } \\
\text { Dificultad de visualización de la región pineal y cuadrigémina }\end{array}$ \\
\hline $\begin{array}{l}\text { Transcortical } \\
\text { posterior (ATCP) }\end{array}$ & $\begin{array}{l}\text { Sin lesión de la vía visual y área del lenguaje } \\
\text { Adecuada visualización de la porción contralateral del III ventrículo } \\
\text { Posible exposición completa del III ventrículo }\end{array}$ & $\begin{array}{l}\text { Precisa hidrocefalia } \\
\text { Requiere corticotomía parietal } \\
\text { Riesgo de daño del fórnix y sistema venoso profundo } \\
\text { Dificultad de visualización de la porción ipsilateral del III ventrículo } \\
\text { Identificación de la región pineal y cuadrigémina complicada }\end{array}$ \\
\hline
\end{tabular}

te de la dosis; el trabajo de Schild et al demostró una tasa de recidiva local inferior en aquellos pacientes tratados con dosis superiores a 50 Gy en comparación con aquéllos que recibieron dosis inferiores [6]. Los pineocitomas extirpados completamente no precisan tratamiento con radioterapia; algunos autores apuestan por el tratamiento con radioterapia de pineocitomas más indiferenciados y aquéllos con un comportamiento más próximo a los pineoblastomas [7]. Asimismo, se han publicado trabajos sobre el papel de la radiocirugía en el tratamiento de pineocitomas; a pesar de que la experiencia es limitada en la actualidad, los resultados son bastante prometedores [21].

El protocolo de quimioterapia aplicada en el tratamiento de los pineoblastomas es similar al empleado habitualmente en el tratamiento de los meduloblastomas (vincristina, carmustina, procarbacina, cisplatino, ciclofosfamida), debido a la similitud de ambos tumores en su comportamiento y tendencia a diseminarse en la región leptomeníngea. En niños pequeños, el tratamiento quimioterápico puede retrasar la administración de radioterapia. No obstante, los mejores resultados parecen obtenerse de la administración conjunta de quimioterapia y radioterapia en estos tumores [22]. Por este motivo, actualmente se desarrollan protocolos de quimioterapia mieloablativa y trasplante hema- topoyético autólogo, con resultados alentadores en la supervivencia de estos pacientes [23].

Los germinomas son extremadamente sensibles a la radioterapia. Las series publicadas recientemente establecen un período de supervivencia libre de enfermedad superior al $90 \%$ en los casos de germinomas puros localizados [24]. Tradicionalmente, la dosis de irradiación tumoral ha sido de 50-54 Gy, pero los trabajos recientes han tratado de disminuir la dosis de radioterapia óptima y de aminorar el volumen de irradiación, para minimizar los efectos secundarios y obviar la necesidad de radioterapia craneoespinal en niños pequeños, sin merma de los buenos resultados de supervivencia. Por este motivo, debido a la alta quimiosensibilidad de los germinomas, se ha asociado la quimioterapia al tratamiento con radioterapia [25].

Aunque la irradiación craneoespinal no está indicada en pacientes con germinomas localizados, los datos obtenidos de la bibliografía sí sugieren incluir el sistema ventricular en el volumen de irradiación, con el fin de reducir la tasa de recidiva tumoral [26]. La irradiación craneoespinal sólo se recomienda en pacientes con diseminación leptomeníngea demostrada en los estudios de RM o con los hallazgos en el LCR.

Los germinomas presentan una buena respuesta a determinados fármacos quimioterápicos (deriva- 
dos del platino), con los que se logran unas cifras de remisión parcial y completa cercanas al 100\% de los casos, permitiendo obviar la irradiación espinal en pacientes afectos de germinomas localizados y reducir la dosis total de radioterapia intracraneal. Sin embargo, la administración aislada de quimioterapia ha mostrado un empeoramiento en las cifras de supervivencia en estos pacientes, por lo que, actualmente, no se recomienda prescindir del tratamiento con radioterapia [26,27].

Los tumores no germinomatosos son menos frecuentes y más variados que los anteriores, por lo que los datos disponibles proceden de series pequeñas, con resultados dispares y difíciles de interpretar. El papel de la irradiación craneoespinal en niños con tumores no germinomatosos localizados no está claro [28,29], aunque la administración de radioterapia holocraneal sí parece ser necesaria.

Múltiples estudios han demostrado que la administración de quimioterapia previamente a la irradiación de los pacientes con tumores no germinomatosos ha incrementado las tasas de supervivencia hasta un 60-70\% [28]. La mayoría de protocolos emplea derivados del platino (cisplatino o carboplatino) y etopósido, asociando ciclofosfamida en algunas ocasiones. La elevación de las cifras de alfafetoproteína constituye un factor pronóstico, ya que se relaciona con la supervivencia de estos pacientes. Los pacientes con niveles normales de alfafetoproteína tienen una supervivencia cercana al $80 \%$, valores de alfafetoproteína de 100-1.000 $\mu \mathrm{g} / \mathrm{L}$ se asocian con una tasa de supervivencia del $60 \%$, y valores superiores a $1.000 \mu \mathrm{g} / \mathrm{L}$ presentan una supervivencia muy baja, inferior al 33\% [24]. No obstante, los valores de bhCG no parecen influir en la supervivencia de los pacientes.

\section{Pronóstico}

De acuerdo con Matsutani, los TCG se pueden clasificar en tres grupos atendiendo a su pronóstico. Los germinomas y los teratomas maduros se incluyen en el grupo considerado de buen pronóstico, con una supervivencia global por encima del $90 \%$; el tumor del seno endodérmico, coriocarcinoma, carcinoma embrionario y tumores de células no germinales mixtos se encuentran dentro del grupo con mal pronóstico, el cual conlleva una supervivencia global media del 40\% [30].

Los TPP tienen como principales factores pronósticos el tipo histológico de la lesión y la existencia de enfermedad diseminada. Los tumores con diseminación leptomeníngea o metástasis espinales tienen un mal pronóstico independientemente del tratamiento recibido [31]. No obstante, estos tumores son infrecuentes y, por tanto, la información actualmente es limitada para proporcionar datos definitivos respecto al pronóstico. En los cuatro trabajos publicados hasta la fecha, 15 de los 21 pacientes presentaron recidiva tumoral incluso después de lograrse una resección completa [32]. Actualmente, el único factor pronóstico de los TPP identificado en la bibliografía es una resección quirúrgica completa de la lesión [33].

En conclusión, los tumores que afectan a la región pineal constituyen un grupo heterogéneo de lesiones con una amplia variedad de rasgos clinicopatológicos, y un pronóstico muy diferente. No obstante, los TRP comparten determinadas similitudes referentes a su manejo quirúrgico y oncológico adyuvante, a consecuencia de la localización específica en la región pineal. En la última década se ha obtenido una mejor resultado en cuanto al pronóstico y la supervivencia de los pacientes afectos de TRP, que podría ser el resultado de las mejoras introducidas en el campo de la neurorradiología, la técnica microquirúrgica, el tratamiento oncológico con radioterapia y/o quimioterapia, o bien una conjunción de todos estos factores. Los germinomas y los TPP representan los tumores más frecuentes en general, y su incidencia es significativamente mayor durante la edad infantil. Los TCG presentan los mejores resultados y una supervivencia mayor; la resección tumoral completa de estos tumores no modifica el pronóstico del paciente, aunque sí se ha descrito un papel importante de la radioterapia en este sentido.

Bibliografía

1. Bruce JN, Kennedy B. Pineal tumors. eMedicine. URL: http:// emedicine.medscape.com/article/249945-overview. [12.06.2010].

2. Villarejo F, Madero L. Tumores del sistema nervioso central en niños. Madrid: Ergon; 2007.

3. Bruce JN. Supracerebellar approach to pineal region lesions. In Sekhar LN, Fessler RG, eds. Atlas of neurosurgical techniques. Brain. 1 ed. New York: Thieme; 2006. p. 549-55.

4. Al-Hussaini M, Sultan I, Abuirmileh N, Jaradat I, Qaddoumi I. Pineal gland tumors: experience from the SEER database. J Neurooncol 2009; 94: 351-8.

5. Central Brain Tumor Registry of the United States (CBTRUS). Statistical report: primary brain tumors in the United States in 2004-2005. URL: http://www.cbtrus.org.

6. Schild SE, Scheithauer BW, Schomberg PJ, Hook CC, Kelly PJ, Frick L, et al. Pineal parenchymal tumors. Clinical, pathologic and therapeutic aspects. Cancer 1993; 72: 870-80.

7. Lassman AB, Bruce JN, Fetell MR. Metastases to the pineal gland. Neurology 2006; 67: 1303-4.

8. Tumours of the pineal region. In Louis DN, Ohgaki H, Wiestler OD, Cavenee, WK, Burger PC, Jouvet A, et al, eds. WHO classification of tumours of the central nervous system. Albany, NY: WHO Publication Center; 2007.

9. Balmaceda C, Heller G, Rosenblum M, Díez B, Villablanca JG, 
Kellie S, et al. Chemotherapy without irradiation -a novel approach for newly diagnosed central nervous system germ cell tumors: results of an international cooperative trial. J Clin Oncol 1996; 14: 2908-15.

10. Chiechi MV, Smirniotopoulos JG, Mena H. Pineal parenchymal tumors: CT and MR features. J Comput Assist Tomogr 1995; 19: 509-17.

11. Abay EO, Laws ER, Grado GL, Bruckman JE, Forbes GS, Gómez MR, et al. Pineal tumors in children and adolescents. Treatment by CSF shunting and radiotherapy. J Neurosurg 1981; 55: 889-95.

12. Bruce JN, Stein BM. Surgical management of pineal region tumors. Acta Neurochir (Wien) 1995; 134: 130-5.

13. Edwards MSB, Hudgins RJ, Wilson CB, Levin VA, Wara WM. Pineal region tumors in children. J Neurosurg 1988; 68: 689-97.

14. Herrmann H, Westphal M, Winkler K, Laas RW, Schulte FJ. Treatment of nongerminomatous germ-cell tumors of the pineal region. Neurosurgery 1994; 34: 524-9.

15. Reddy AT, Wellons JC, Allen JC, Fiveash JB, Abdullatif H, Braune KW, et al. Refining the staging evaluation of pineal region germinoma using neuroendoscopy and the presence of preoperative diabetes insipidus. Neuro Oncol 2004; 6: 127-33.

16. Kreth FW, Schätz CR, Pagenstecher A, Faist M, Volk B, Ostertag CB, et al. Stereotactic management of lesions of the pineal region. Neurosurgery 1996; 39: 280-9.

17. Regis J, Bouillot P, Rouby-Volot F, Figarella-Branger D, Dufour H, Peragut JC. Pineal region tumors and the role of stereotactic biopsy: review of the mortality, morbidity and diagnostic rates in 370 cases. Neurosurgery 1996; 39: 907-12.

18. Popovic EA, Kelly PJ. Stereotactic procedures for lesions of the pineal region. Mayo Clin Proc 1993; 68: 695-70.

19. Dempsey PK, Lunsford LD. Stereotactic radiosurgery for pineal region tumors. Neurosurg Clin N Am 1992; 3: 245-53.

20. Kondziolka D, Hadjipanayis CG, Flickinger JC, Lunsford LD. The role of radiosurgery for the treatment of pineal parenchymal tumors. Neurosurgery 2002; 51: 880-9.

21. Jonhston DL, Keene DL, Lafay-Cousin L, Steinbok P, Sung L, Carret AS, et al. Supratentorial primitive neuroectodermal tumors: a Canadian pediatric brain tumor consortium report. J Neurooncol 2008; 86: 101-8.
22. Gururangan S, McLaughlin C, Quinn J, Rich J, Reardon D, Halperin EC. High-dose chemotherapy with autologous stem-cell rescue in children and adults with newly diagnosed pineoblastomas. J Clin Oncol 2003; 21: 2187-91.

23. Borg M. Germ cell tumours of the central nervous system in children-controversies in radiotherapy. Med Pediatr Oncol 2003; 40: 367.

24. Rogers SJ, Mosleh-Shirazi MA, Saran FH. Radiotherapy of localised intracranial germinoma: time to sever historical ties? Lancet Oncol 2005; 5: 509-19.

25. Allen JC, DaRosso RC, Donahue B, Nirenberg A. A phase II trial of preirradiation carboplatin in newly diagnosed germinoma of the central nervous system. Cancer 1994; 74: 940-4.

26. Douglas JG, Rockhill JK, Olson JM, Ellenbogen RG, Geyer JR. Cisplatin-based chemotherapy followed by focal, reduceddose irradiation for pediatric primary central nervous system germinomas. J Pediatr Hematol Oncol 2006; 28: 36-9.

27. Calaminus G, Bamberg M, Baranzelli MC, Benoit Y, Di Montezemolo LC, Fossati-Bellani F. Intracranial germ cell tumors: a comprehensive update of the European data. Neuropediatrics 1994; 25: 26-32.

28. Matsutani M. Combined chemotherapy and radiation therapy for CNS germ cell tumors. The Japanese experience. J Neurooncol 2001: 54: 311-6.

29. Guerrero-Vázquez S, Armesto-Pérez V, Macía-Suárez D, Brañas-Fernández FM. Germinoma pineal y supraselar sincrónico: a propósito de un caso. Rev Neurol 2008; 46: 411-5.

30. Chang SM, Lillis-Hearne PK, Larson DA, Wara M, Bollen AW, Prados MD. Pineoblastoma in adults. Neurosurgery 1995; 37: 383-90.

31. Fevre-Montange M, Hasselblatt M, Figarell-Branger D, Chauveinc L, Champier J, Saint-Pierre G, et al. Prognosis and histopathologic features in papillary tumors of the pineal region: a retrospective multicenter study of 31 cases. J Neuropathol Exp Neurol 2006; 65: 1004-11.

32. Sato TS, Kirby PA, Buatti JM, Moritani T. Papillary tumor of the pineal region: report of a rapidly progressive tumor with possible multicentric origin. Pediatr Radiol 2009; 39: 188-90.

33. López-Muñoz F, Marín F, Álamo C. El devenir histórico de la glándula pineal: II. De sede del alma a órgano neuroendocrino. Rev Neurol 2010; 50: 117-25.

\section{Tumours in the pineal region in the paediatric age. Reports of 23 cases and a review of the literature}

Introduction. Tumours in the pineal region are located at a meeting point of several neurovascular structures that are difficult to reach surgically and for which the possibility of resection is limited; as a result the management of these lesions usually requires associated adjunctive treatment with radiotherapy and/or chemotherapy.

Patients and methods. This study is a retrospective analysis of the epidemiological, clinical, neuroimaging and pathological characteristics of 23 patients with tumours in the pineal region who were treated between the years 1997 and 2010 in the Hospital Infantil Niño Jesús. The factors involved in the prognosis of this cohort following surgical or adjunctive treatment are also discussed.

Results. Subjects included in the study were 6 girls and 17 boys with ages ranging from 4 months to 18 years. It was found that the initial symptoms in $95 \%$ of the patients were signs of acute or subacute hydrocephalus, which required the placement of a ventriculoperitoneal shunt (82\%). A histological sample of the tumour tissue was collected in all cases. Biopsy samples were taken in the case of five patients and 18 underwent surgery involving a craniotomy. Germinoma (eight cases) and mature teratoma (one case) were the tumours with the longest survival times; non-germinomatous tumours (three cases), those of the pineal parenchyma (four cases) and gliomas (five cases) presented the highest rates of recurrence and a poorer prognosis.

Conclusions. The study of tumour markers can be used to guide the diagnosis of certain tumours of the pineal region. At present, the recommended procedure involves taking a histological sample of the tumour in order to establish an accurate diagnosis and a specific oncological treatment.

Key words. Astrocytoma. Germinoma. Pineal gland. Pineal region. Pineoblastoma. Pineocytoma. Tumour. 